\title{
L'Homme et la Loire
}

\author{
J. Bethemont \\ Université Jean Monnet, Saint-Etienne
}

J.G. Wasson

CEMAGREF, Lyon

\begin{abstract}
Le bassin de la Loire couvre approximativement le cinquième du territoire français, soit $117054 \mathrm{~km}^{2}$. occupés par 7,8 millions d'habitants. Si le débit spécifique du fleuve est relativement modeste $\left(7,1 \mathrm{l} / \mathrm{km}^{2}\right)$, son module mesuré dans la partie inférieure de son cours à Montjean n'en est pas moins important : $835 \mathrm{~m}^{3} / \mathrm{s}$, soit près de $40 \mathrm{~km}^{3} / \mathrm{an}$. Sur cette base et compte tenu d'une charge humaine somme toute modeste ( 66 habitants par $\mathrm{km}^{2}$ ), il semble de prime abord que les problèmes liés à la gestion de la ressource en eau devraient être de facile résolution, n'était une impressionnante série de données paradoxales : ce fleuve atlantique s'avère méditerranéen tant par ses extrêmes, étiages et crues (de $20 \mathrm{~m}^{3} / \mathrm{s}$ à $9800 \mathrm{~m}^{3} / \mathrm{s}$ dans le secteur de Briare) que par son coefficient d'immodération de 1 à $6\left(256 \mathrm{~m}^{3} / \mathrm{s}\right.$ en août et $1520 \mathrm{~m}^{3} / \mathrm{s}$ en février à Montjean), ce qui ne l'empêche pas de subir des embâcles sévères ; ce fleuve inconstant fut un temps et jusqu'à l'avènement du chemin de fer, la principale artère navigable en France, avec un trafic annuel de 300000 tonnes, supérieur à ceux de la Seine ou du Rhône, avant d'être totalement délaissé ; ce fleuve que l'on dit être «le dernier fleuve sauvage d'Europe», est également celui qui a été le plus anciennement et le plus radicalement transformé par l'action humaine, ce qui n'est pas incompatible avec une réelle biodiversité, elle -même menacée tant par la montée des usages de l'eau que par les modalités de ces usages.
\end{abstract}

A partir de ces données contradictoires dont la liste pourrait être plus longue, l'analyse des relations entre les hommes et le fleuve' ${ }^{1}$, peut se ramener à trois thèmes portant sur l'aménagement du cours, la gestion de la ressource en eau et la structuration de l'espace par l'eau. De l'un à l'autre de ces thèmes, se pose le problème de l'impact de ces activités sur l'écologie du système Loire.

\section{I L'AMÉNAGEMENT DU COURS}

Si l'on considère que trois types d'activités concourent sous des formes diverses à l'altération des lits fluviaux, la navigation, la production d'énergie et l'extraction de matériaux, et si l'on ajoute à ce décompte la protection des champs d'inondation, le «dernier fleuve sauvage de France» est incontestablement le fleuve le plus anciennement et le plus continûment aménagé des fleuves français. Un fleuve coupé par des barrages, corseté par des digues et exploité comme une vaste carrière.

\subsection{Des moulins usiniers aux ouvrages régulateurs}

Le projet avorté du Serre-de-la-Fare qui était censé mettre à mal tout l'équilibre écologique du fleuve ne constituait à tout prendre, que le dernier en date d'une succession d'aménagements réalisés à des fins diverses et dont les effets d'impact ont été non moins divers.

\subsubsection{Trois générations d’ouvrages}

Les moulins constituent une première génération d'ouvrages et leur recension sur la carte de Cassini montre qu'en termes de statistique, les affluents de la Loire comptaient un moulin pour un peu plus d'un kilomètre linéaire. Beaucoup ayant été détruits, on n'en compte plus maintenant qu'un pour trois kilomètres. Des moulins de toute sorte, au fil de l'eau (barrage-dérivation-moulin-bief de restitution) ou sur étang, avec ou sans pertuis, écluse ou vanne de navigation. Au niveau le plus modeste, se situent les moulins à grain dont le premier dans l'ordre spatial se trouve à moins d'un kilomètre à vol d'oiseau des sources de la Loire ; au niveau le plus élaboré de véritables usines, implantées à l'époque proto-industrielle comme les moulins de Brives-Charensac ou de Richard-le-Bas mais dont certaines ont passé l'épreuve du temps en restant fonctionnelles. Elles animent encore de petites cellules d'activité (de la meunerie à la galvanoplastie), alors que d'autres ont été équipées en micro-centrales.

Les techniques modernes de la houille blanche n'ont pas beaucoup ajouté à ce tableau, bien qu'on dénombre 11 installations EDF sur l'Allier et la Loire supérieure (dont la dérivation d'Issarlès-Montpezat vers l'Ardèche), 15 sur la Loire à l'aval de l'Ance et sur l'Allier à l'aval de l'Alagnon, 27 sur la Vienne et la Creuse. La productibilité de

\footnotetext{
I L'essentiel des problèmes que pose un aménagement intégré du bassin de la Loire ont été posés dans le cadre d'un rapport intitulé "Appproche écosystémique du bassin de la Loire, éléments pour l'élaboration des orientations fondamentales de gestion" et réalisé en 1993 conjointement par le CEMAGREF (G. Wasson)et le CNRS (Jacques Bethemont, J.N. Degorce, B. Dupuis et T. Joliveau) pour le compte du ministère de l'environnement.
} 
l'ensemble est modeste, soit $1660 \mathrm{GWh}$ à comparer avec les $2000 \mathrm{GWh}$ du seul équipement de Donzère-Mondragon.

Le barrage de Villerest relève d'une troisième génération d'ouvrages destinés en première analyse à la régulation hydraulique. En fait ce barrage implanté à l'amont de Roanne, aligne un productible de $160 \mathrm{GWh}$ mais sa construction réalisée à l'initiative de l'EPALA répond surtout au besoin de protection contre les crues, d'où la complexité d'une gestion qui vise simultanément au stockage des débits de pointe et au soutien des étiages. Exercice difficile puisque les calendriers de probabilité des événements extrêmes, étiages et crues, sont à peu près superposables au printemps et surtout à l'automne. La finalité et la gestion de Naussac sont plus simples, puisque cet ouvrage implanté en marge de l'Allier à l'amont de Langogne, stocke par pompage des eaux prélevées en période de fort débit et relachées en soutien des étiages, soit 781 millions de $\mathrm{m}^{3}$ représentant une hauteur d'eau rapportée à la superficie du bassin de 6,5 mm équivalant à $2,75 \%$ de l'écoulement à l'échelle du bassin. Pourrait également contribuer à la régulation hydraulique, l'ouvrage écrêteur de crue dont la mise en place est projetée sur le site du Veurdre dans le cours terminal de l'Allier.

\subsubsection{Des ouvrages contestés}

Le projet du Serre-de-la-Fare défini au lendemain de la crue catastrophique de septembre 1980 (8 morts et 500 MF de dégats) ne différait pas sensiblement de l'ouvrage de Villerest dans sa logique de gestion : écrêtement des crues et soutien des étiages. Il tombait donc sous le coup de cette même critique qui soutient qu'un barrage peut difficilement être à la fois vide pour accueillir les crues d'automne et plein pour soutenir les étiages de fin d'été. Il lui fut surtout reproché d'ennoyer un site de gorge rare, qui abritait une faune et une flore de qualité exceptionnelle (à noter que l'annonce de tout projet d'aménagement fait éclore les flores rares et se multiplier les espèces animales en danger). Les manipulations d'opinion firent le reste. De façon plus sérieuse, la crise des aménagements ligériens a contribué à la définition d'un corps de doctrine ${ }^{2}$ sur les effets d'impacts irréversibles des grands ouvrages hydrauliques : altération de la qualité de l'eau par désoxygénation, piégeage des sédiments et rupture de la continuité aval-amont des chaînes biologiques, le problème des anadromes s'avérant particulièrement grave. A quoi s'ajoute le risque de caléfaction mesuré en aval des centrales nucléaires actuelles ou en cours d'achèvement (Civaux alimenté par la retenue de Vassivière) dont la demande en eau de refroidissement est pour partie à l'origine des retenues.

\footnotetext{
2 L'ouvrage collectif édité par C. Amoros et G. Petts, «Hydrosystèmes fluviaux», Paris, Masson, 1993, 300 p, donne, sous la plume de J.P. Bravard. une mise au point synthétique sur les problèmes d'impact liés aux grands aménagements hydrauliques.

${ }^{3}$ Problème étudié ave beaucoup de fougue par O. Balabanian et G. Bouet. "L'eau et la maîtrise de l'eau en Limousin». Naves, 1989, eds. "Les Monédières, 296 p.
}

Au-delà du cas particulier du Serre-de-la-Fare, la remise en cause des pratiques d'aménagement s'est étendue à l'ensemble des ouvrages ligériens avec, pour «fil conducteur», le saumon, espèce emblématique de la Loire. Sont particuliérement visés Poutès-Monistrol sur l'Allier et Maisons-Rouges au confluent de la Creuse et de la Vienne. Il s'agit d'ouvrages modestes dont l'appport énergétique est négligeable eu égard à leur impact. Ce sont également des ouvrages en fin de concession dont l'arasement programmé permettra aux saumons d'accéder aux frayères de l'Allier (sous réserve d'un aménagement dans la traversée de Vichy) et des affluents de la Vienne, notamment la Gartempe.

Reste le problème des ouvrages de petite hydraulique ${ }^{3}$. Il est intéressant sur ce point de noter, à titre d'exemple, le cas des ouvrages implantés sur la Gartempe : il fut un temps où les saumons les franchissaient en nombre et où la Gartempe fournissait en abondance - réglementée - les Halles de Paris. Les remontées s'interrompirent du jour au lendemain quand le barrage de Maisons-Rouges fut mis en eau. Qu'en sera-til lorsque cet obstacle disparaîtra ? Le témoignage des vieux meuniers comme l'analyse des archives consacrées aux pêches laissent entendre, en tout état de cause, que les petits ouvrages dont la puissance de commandement excédait rarement le mètre, ne constituaient pas un obstacle au continuum spatial de l'arbre fluvial. Mais alors, à quoi bon ces passes et ces échelles à poissons que l'on aménage systématiquement au droit des barrages et que n'emprunte en tout état de cause aucun saumon?

\subsection{Des levées de la Loire à la Loire chenalisée}

De Decize à Nantes, sur 530 kilomètres, la Loire est enserrée de façon à peu près continue par des digues qui dominent de plus de 7 mètres son lit ordinaire et dont l'assise atteint près de 30 mètres pour une largeur de 12 mètres au couronnement. Une entreprise pharaonique dont l'ampleur étonne, surtout lorsque l'on songe qu'elle a été menée à bien sans outillage autre que manuel et qu'il s'est écoulé plus de sept siècles entre les premiers ouvrages du XIIème et le quasi achèvement des travaux dans l'état actuel, à la fin du siècle dernier. L'ampleur de ce délai laisse entendre que les travaux ne furent jamais menés de façon continue, sauf peut-être à l'époque de Colbert, et les relevés de chantier montrent que les ouvrages furent repris, rehaussés et élargis à diverses époques. En l'état, l'ouvrage répond à des finalités diverses, les unes obsolètes les autres encore fonctionnelles.

\subsubsection{Ce fleuve qui fut navigable}

A l'état naturel, le lit du fleuve était frangé, au contact du lit majeur et de la plaine d'inondation, par une suite discontinue de bourrelets fluviaux formés par le dépot de matériaux grossiers lors des phases de débordement. C'est sans doute de façon très empirique et progressive que ces bourrelets ou levées ont été reliés entre eux et rehaussés de façon à créer le système actuel. Sans doute s'agissait-il initialement, c'est-à-dire à l'époque des ducs d'Anjou, de réaliser des 
chaussées moins submersibles que les terres basses avoisinantes, chaussées qui furent initialement implantées en rive droite entre le fleuve et l'Authion.

De façon incidente, ces premières levées eurent pour effet de protéger de façon plus ou moins sûre les villes et les champs du Val. Mais leur effet le plus remarquable fut la fixation dans un lit stable, d'un fleuve jusqu'alors instable du fait de sa propension au méandrage ou au tressage. L'intérêt passa donc dès le XVème siècle, des chaussées ou de la protection des terres, à l'amélioration des conditions de la navigation. C'est également à cette fin que furent implantées dans le lit fluvial de longues digues basses, les duies destinées à concentrer les eaux moyennes et basses dans un chenal unique. Les résultats étaient loin d'être parfaits et l'intensité relative d'un trafic souvent interrompu par les écarts du fleuve, tenait moins aux conditions matérielles de la navigation qu'au mauvais état des routes et aux intérêts en jeu : le port de Nantes commandait le précieux trafic des Iles et, tout compte fait, la Loire - le vent d'ouest aidant - était plus navigable à la remontée que la Seine sur laquelle le trafic montant était entravé par la disposition des méandres. D'où le recours à la Loire et le creusement pécoce du canal de Briare qui assurait la jonction avec le Loing et la Seine. L'animation du commerce de Loire s'étendait très loin vers l'amont puisque les têtes de navigation se situaient à Saint-Rambert sur la Loire et au confluent de la Dore sur l'Allier. A la descente, le trafic portait sur du bois, du charbon, du fer, des articles de forge et des arts du feu ; à la remontée, des produits coloniaux, des produits textiles, des vins. En dépit de multiples aménagements comme le canal latéral de la Loire, le canal du Berry et le canal du Nivernais, la navigation ligérienne a tôt succombé à la concurrence de la voie ferrée et elle s'est pratiquement éteinte avant la fin du XIX ${ }^{\text {ème }}$ siècle. Elle n'en est pas moins à l'origine d'activités aussi diverses que les vins de Touraine et d'Anjou, les industries mécaniques de Bourges, Vierzon, Montluçon ou les porcelaines du Berry pour n'en citer que quelques unes.

Tout cela se passait bien avant que les règles de l'aménagement fluvial n'eussent été définies par Vasarely et Girardon, de sorte que les levées ne constituent en fait qu'un ensemble disparate où la largeur du lit entre digues ne répond pas toujours à une conception d'aménagement logique : pour une largeur moyenne d'environ mille mètres au niveau d'Orléans, la largeur entre berges varie d'une section à une autre, celles-ci sont parfois mal raccordées entre elles et surtout elles se resserrent à moins de 300 mètres au droit des ponts urbains : de là des reflux dangereux en période de crue et des risques d'embacles en période de grands froids.

\subsubsection{Un cours inférieur déstabilisé}

A l'état naturel et jusqu'à la fin du XVIII è̀me siècle, l'estuaire se présentait sous forme d'un ensemble de chenaux séparés par des îles et bordé par des vasières dont l'intérêt écologique n'a été que tardivement perçu. Par la suite, après que le creusement d'un canal latéral en rive gauche (canal de la Martinière) se soit avéré insuffisant, les eaux ont été progressivement concentrées dans un chenal unique dont la profondeur a été portée à 12 mètres au niveau de Cordemais et 4 mètres au niveau du Pellerin. Simultanément, toute une série d'implantations lourdes ont occupé les berges, principalement en rive droite, réduisant à peu de chose, les vasières et leurs biotopes.

Ces travaux portuaires ont eu pour corollaire, dans les années 60 , l'aménagement d'un chenal navigable entre Nantes et Bouchemaine. Le trafic, assuré par des barges de $600 \mathrm{tpl}$, consistait pour l'essentiel en matériaux de construction et en produits pétroliers et n'a jamais dépassé le cap des 500000 tonnes. C'était évidemment très peu pour l'hinterland supposé d'un port dont le trafic atteint 25 millions de tonnes de sorte que la voie fluviale a fini par être déclassée. Entre temps, le fleuve a été aménagé par concentration des eaux dans un chenal unique fixé par des épis plongeants. Toujours aux fins d'amélioration, le seuil de Bellevue, dernier affleurement rocheux situé juste en amont de Nantes a été arasé. Une fois passé le temps de la Loire navigable, restent les impacts de ces grands travaux, essentiellement la remontée de la langue salée et du bouchon vaseux, bien au-delà du seuil de Bellevue et jusqu'au niveau d'Ancenis avec, pour effet second, l'envasement des puits de captage de l'agglomération nantaise, qu'il a fallu reporter vers l'amont.

\subsubsection{Les levées : une protection relative ou illusoire}

A défaut de rendre le fleuve navigable, les levées ont permis de diversifier les sites d'habitat humain jusqu'alors confinés au pied des côteaux, aux points hauts des levées originelles et aux montilles ou buttes sableuses qui parsèment le Val. Est-ce à dire qu'elles assurent une protection efficace aux quelque 200000 personnes qui vivent maintenant dans ce qui serait des zones de fort débit en cas de rupture des levées ? Il est certain qu'on n'a plus revu sur la Loire des débits comparables à ceux des hautes eaux de 1856, date à laquelle les levées furent rompues par 16 brèches en rive droite, 20 en rive gauche et le Val inondé dans sa totalité. Le Val avait déjà été noyé en 1608, 1710$1711,1733,1846,1856$ et le sera à nouveau en 1866. En 1856 , et en dépit des brèches amont, les eaux atteignirent la cote de 7,52 mètres à Tours, pour une hauteur de 7 mètres à l'arasement des digues. L'état de celles-ci n'a pas beaucoup changé depuis cette date et rien n'interdit de penser que de futures catastrophes entrent dans l'ordre des probabilités, puisque l'occupation sûre des lieux n'est garantie que pour des débits inférieurs à $6000 \mathrm{~m}^{3} / \mathrm{s}$. Encore faut-il observer que les secteurs inférieurs des vals sont envahis par des remous, au niveau des confluents en période de hautes eaux.

Rappelons qu'après la crue de 1856, l'ingénieur Comoy avait établi un plan de protection prévoyant la construction de 85 retenues sur les cours supérieurs du fleuve et de ses affluents, avec une capacité de stokage évaluée à 500 millions de mètres cubes. A défaut, il proposait de faire la part de l'eau en ménageant des points de rupture bien choisis sur des levées commandant des vals peu ou pas occupés de 
façon permanente et aménagés en déversoirs. Au nombre de 20 , ces ouvrages devaient permettre la sauvegarde des villes au prix de l'ennoiement de la moitié des surfaces inondables sises en arrière des levées (44 000 hectares sur 96000 ). Le temps passant, le sentiment de l'urgence s'effaçant et l'hostilité des riverains menacés par ces contraintes aidant, 7 seulement de ces ouvrages ont été réalisés. Le problème de la protection d'un Val toujours plus occupé reste donc presque entier, le seul ouvrage de Villerest ne constituant pas une réponse à la mesure du risque, non plus que la construction programmée de l'ouvrage écrêteur du Veurdre.

\subsubsection{La référence aux polders}

La réponse la plus radicale à l'aléa fluvial, en l'état des techniques, pourrait bien consister en une poldérisation des vals les plus urbanisés où se concentrent l'essentiel des risques. Il existe dans ce domaine, une référence concrète correspondant au Val d'Authion, proche d'Angers. Cette vallée affluente en rive droite, faiblement encaissée entre le côteau et la levée, est drainée de façon naturelle en basses eaux mais son tronçon terminal est équipé au niveau du confluent, d'une écluse fermée en cas de crue et flanquée d'une station de pompage qui assure l'exhaure. Reste à savoir si ce modèle répond aux besoins urbains, puisque les aménageurs ont respecté une zone basse marécageuse et une zone de contrainte (prairie naturelle) qui permet une certaine régulation des flux hydriques dans un espace qui reste essentiellement rural.

\subsection{Le fleuve comme carrière}

Il a fallu l'effondrement du pont de Tours en avril 1978, pour que soit prise la mesure de cet impact trop diffus pour être convenablement évalué, que constitue le prélèvement de matériaux dans le lit du fleuve. Une pratique traditionnelle certes, mais les extractions annuelles mesurées entre le Bec d'Allier et Nantes sont passées de 150000 tonnes en 1860 à un million en 1950 pour culminer à 10 millions en 1978 , ce qui représente un volume extrait de $300 \mathrm{~m}^{3}$ par mètre de linéaire. D'où un enfoncement du lit, variable mais pouvant atteindre de 1 à 3 mètres entre l'Orléanais et l'Anjou. L'ampleur du phénomène ayant été justement évaluée... et les grands travaux routiers, grands consommateurs de sables et graviers étant momentanément achevés, on a décidé sauf exception - d'arrêter les extractions entre les levées à dater de 1992.

N'importe, le mal est fait et ses conséquences, outre l'enfoncement du lit, se feront sentir longtemps, d'autant que la puissance de la nappe alluviale avait été surévaluée. Sa minceur était masquée par la mobilité des bancs qui comblaient les fouilles mais actuellement et faute de matériau de renouvellement, le substrat rocheux est souvent mis à nu. S'ensuit naturellement une reprise d'érosion par secteurs localisés mais, en l'absence de matériaux charriés depuis l'amont, la recharge se fait par sapement latéral des berges. Outre le risque de déstabilisation des levées qui résulte de ce processus, la dynamique fluviale est affectée par la substitution aux sables grossiers (sables de jard) qui tapissaient le lit, de limons fins difficilement mobilisables après dépot et à fort pouvoir colmatant. De la conjonction de ces diverses altérations de l'hydrosystème, résulte une déconnexion appauvrissante entre le cours principal, des bras secondaires moins souvent visités et des îles plus hautes, plus sèches et plus exposées à l'érosion.

Le prélèvement de matériaux en dehors du lit mineur, pratique courante en amont du Val endigué, ne constitue pas une solution de rechange satisfaisante, ne fut-ce que parce qu'elle contribue à l'abaissement des nappes phréatiques. Du moins se prête-t-elle à des actions compensatoires comme le remarquable réaménagement des gravières et de la ripisylve de la région stéphanoise, dans le cadre de l'Ecopole du Forez.

\subsection{Le temps est galant homme}

La liste des interventions humaines sur la Loire est évidemment fort longue et leur bilan s'inscrit sur un registre globalement négatif. D'où vient alors que ce «fleuve sauvage” passe pour un modèle intangible de bon fonctionnement écologique ? La diversité des biotopes ligériens étant un fait avéré, reste à s'interroger sur ce qu'on peut appeler le paradoxe ligérien. Trois ordres de considérations pourraient être développés à ce sujet.

Il apparaît tout d'abord que beaucoup d'aménagements anciens comme les barrages des cours supérieurs étaient de taille trop modeste pour être réellement et durablement offensants. De même, les prélèvements de matériaux dans le lit ne dépassaient pas la capacité de renouvellement annuel des nappes d'alluvions qui peut être estimé à quelque 200000 tonnes.

Les levées qui constituent le grand aménagement ligérien sont des constructions plus empiriques que rationnelles. Elles ménagent des espaces perdus qui sont autant d'espaces qui, le temps passant, ont été progressivement recouvrés par des chaînes biotiques. Surtout, le vaste espace enserré par les levées n'est accessible que sur des points limités. Il est, pour l'essentiel, soustrait à la plupart des activités anthropiques: on n'y trouve guère que des prises d'eau pour le ravitaillement urbain, quelques cultures sur les îles hautes et, dans le secteur angevin des prairies naturelles et des plantations de peupliers sur lesquelles il y aurait sans doute beaucoup à dire. Pour le reste, le lit du fleuve constitue un espace de récupération pour de nombreuses espèces végétales ou animales.

Ces considérations sur la taille des ouvrages anciens ou sur leur aptitude à intégrer la composante naturelle dans les travaux d'ingénierie amène à s'interroger sur les capacités d'intervention, la taille et la rationalité des travaux actuels : laissent-ils place avec ou sans le secours du temps, à la restauration d'équilibres écologiques qui restent à définir ?

\section{II 둘 LA GESTION DE LA RESSOURCE}

Les problèmes que pose la gestion d'une ressource qui, sans être rare, est à la fois irrégulièrement répartie dans le 
temps et peu régularisable ne seront envisagés ici que de façon globale, en fonction des relations existant entre écosystème et anthroposystème.

\subsection{Les problèmes d'accès et de régulation}

Peut-on parler de déterminisme ? Le fait est que les problèmes d'accès à la ressource se calquent étroitement sur les cartes géologiques et sur celles des formations superficielles. Ce sont en définitive les données naturelles et notamment la relation entre sol et climat qui conditionnent la logique de ce qu'on peut appeler l'espace de l'eau.

Pour plus de la moitié de sa superficie, le bassin ligérien est constitué de roches cristallines, métamorphiques ou schisteuses qui ont en commun leur faible perméabilité et la minceur de leurs formations superficielles. C'est également sur ce type de formation que s'abattent les précipitations efficaces et que se situent les sources et les principales zones de formation des débits, hautes terres du Massif Central, môle morvandiau et Massif Armoricain. Ces régions sont également celles où la densité de drainage est élevée, parfois supérieure à 500 mètres linéaires par kilomètre carré ; des régions où l'eau ruisselle, stagne parfois dans les sagnes ou narses, manque sur de rares planèzes basaltiques mais où, tout compte fait, elle n'est guère stockée d'une séquence pluvieuse à l'autre : ce sont des régions vertes quand il pleut (et il pleut souvent) mais qui connaissent de redoutables sécheresses estivales durant lesquelles beaucoup de sources tarissent. Certaines régions comme les hautes terres du Massif Central subissent cette contrainte, d'autres s'efforcent de pallier par stockage les périodes de carence. C'est en particulier le cas du Limousin où on recense sur le versant ligérien pas moins de 6500 étangs utilisés à de multiples fins, dont 2500 ont une superficie supérieure à 50 hectares.

Aux limites des terrains cristallins et sédimentaires, deux types de relations à l'eau dominent, selon que l'on a affaire aux bassins d'effondrement ou à des formations superficielles imperméables. En théorie, les bassins d'effondrement disposent de bonnes nappes. C'est surtout vrai pour les Limagnes auvergnates favorisées par endroit par leurs sols tourbeux mais bien drainés. C'est également vrai pour le bassin de Roanne, le Val d'Allier et le Val d'Ambert. C'est moins évident pour les petits bassins du Puy et de l'Emblavés dont le substrat sédimentaire est à la fois hétérogène et mince. Le bassin du Forez enfin, offre une hétérogénéité considérable. La Loire facilite l'accès à l'eau grâce au canal du Forez, mais les nappes sont très irrégulières et de vastes secteurs sont couverts par des formations détritiques argileuses ou siliceuses qui servent d'assise aux étangs du Forez. Ces mêmes formations superficielles, restes des nappes d'épandage descendues du Massif Central durant les épisodes secs ou semi-arides du tertiaire ont facilité l'établissement des étangs de la Sologne et de la Brenne.

Entre Massif Central et Massif Armoricain, alternent quelques zones de sols argileux lourds - Charolais, Auxois ou Saosnois - mais pour l'essentiel, ce sont les formations calcaires ou crayeuses qui dominent. La densité de drainage tombe à des valeurs faibles de 1'ordre de 200 mètres linéaires par $\mathrm{km}^{2}$ et de vastes espaces de la table de Beauce peuvent être considérés comme aréiques. Par compensation, ces tables calcaires surmontent des nappes profondes qui constituent une richesse sans doute surexploitée.

Dans le Val, les problèmes liés à la gestion de la ressource sont plus complexes qu'il n'y paraît de prime abord. Non que l'eau soit rare quelques soient les irrégularités du fleuve : la nappe alluviale est proche ; elle est alimentée à la fois par le fleuve (y compris par renards en périodes de hautes eaux) et par les eaux de colature du coteau. Les difficultés tiennent précisément à ces eaux de colature qui forment de petits cours d'eau, boires ou cisses, séparés du fleuve par la levée. Leurs eaux sont fraîches, leurs lits peu profonds et souvent mal drainés à l'état naturel. Ils ont quelque mal à rejoindre le fleuve, alors qu'en période de hautes eaux celui-ci tend à refluer dans leur cours inférieur. Les problèmes de drainage gardent donc une importance primordiale dans l'ensemble du Val et ils peuvent devenir franchement inquiétants dans les zones de confluence urbanisées, la référence à Tours s'imposant sur ce point.

\subsection{L'utilisation de la ressource}

\subsubsection{L'agriculture : progression des surfaces irriguées et problèmes de rejets}

En dépit de séquences sèches calamiteuses (1949 et 1976 pouvant servir de référence), les besoins des plantes en apports d'eau sont moins importants dans le Centre et l'Ouest de la France que sous climat méditerranéen : 2000 $\mathrm{m}^{3}$ par hectare suffisent en année moyenne à la culture du maïs dans la région Centre contre près de 4000 dans la basse vallée du Rhône. Même si le recours à l'irrigation ne constitue qu'une technique d'appoint, celle-ci connaît actuellement une progression considérable. Limitée au secteur primeuriste et à quelques vergers en 1950, elle intéressait 70000 ha en 1979 et 130000 hectares en 1988, chiffre sans doute sous-évalué compte tenu de la nature des équipements. Les installations collectives sont rares en dehors du canal du Forez et les équipements individuels sont la règle à peu près générale. Au pompage dans la nappe du Val, caractéristique de l'Anjou et, dans une moindre mesure des vals de Touraine et de l'Orléanais, est venu s'ajouter le pompage dans les nappes de Beauce qui ont subi un rabattement sensible aggravé au plan écologique par l'accroisssement de la teneur en nitrates de leurs eaux. Autre technique en progression, le pompage dans les cours d'eau, rarement pratiqué en été, le principe étant de pomper en hiver et de stocker l'eau dans des lacs collinaires dont la capacité dépasse souvent 100000 mètres cubes. Les volumes cumulés de ces réserves sont de l'ordre de 25 millions de mètres cubes.

Au plan de la répartition spatiale, trois zones se détachent avec plus du quart de leur S.A.U. sous irrigation : la plaine du Forez, la Beauce y compris la petite Beauce en rive gauche et le Val d'Anjou, en particulier le Val d'Authion ; l'irrigation tient également une place non négligable, soit 
plus de $10 \%$ de la S.A.U. dans la Limagne, le Val de Loire, la région nantaise, la Champagne mancelle et le Seuil de Poitou. Ceci dit, la relation entre l'irrigation et la culture de maïs n'est pas aussi évidente qu'on le croit souvent et l'arrosage intéresse aussi bien des cultures sarclées ou industrielles que des vergers. Ce qui est plus évident en revanche c'est la relation entre irrigation et agriculture intensive. A preuve, l'analyse des revenus agricoles : pour un indice 100 correspondant à la moyenne nationale des revenus nets par exploitation, la totalité des régions irriguées dépasse l'indice 150.

Parmi les autres problèmes agricoles liés à la gestion de l'eau, se situe en bonne position celui des pressions exercées par l'élevage intensif qui intéresse les régions de l'Ouest, Vendée, Mayenne et confins armoricains avec des problèmes de rejets particuliérement évidents dans les régions où existent des nappes phréatiques fragiles comme le seuil du Poitou.

Une vue globale de l'agriculture dans le bassin de la Loire amènerait en définitive à opposer deux types de régions, les unes dynamiques, les autres fragiles. Au titre des régions dynamiques, on comptera outre les Limagnes et le Forez, la totalité du Val de Loire (avec toutefois de sérieuses nuances observables de part et d'autre du coude d'Orléans, la partie aval étant plus dynamique) et les régions de l'Ouest avec une mention spéciale pour la Vendée. Toutes ces régions entretiennent des densités agricoles honorables avec une proportion importante de chefs d'exploitation jeunes. Toutes sont caractérisées par une intensité mesurable aussi bien en termes d'équipement ou de revenus que de pression exercée sur le milieu. Ce sont également des régions où l'herbe recule devant les labours et où les remembrements remettent en cause les paysages patrimoniaux, la disparition du bocage constituant le fait le plus marquant et le plus préoccupant. Au titre des régions fragiles, on comptera les hautes terres du Massif Central et du Morvan, une partie du Bourbonnais, les régions d'étangs, les brandes du Poitou. Toutes ces régions sont caractérisées par la vieillesse de leur population, la faiblessse des revenus agricoles (indice 43 pour le Limousin), le faible niveau d'équipement et le recul des labours devant l'herbe, bientôt suivi du recul de l'herbe devant le bois. A noter toutefois que la déprise humaine va parfois de pair avec l'instauration de nouveaux équilibres qui sont peut-être prometteurs. On se contente sur ce point d'opposer les régions d'élevage intensif aux régions d'élevage sur prairie naturelle avec de faibles charges à l'hectare qui évitent le recours à certaines pratiques discutables.

Ainsi s'opère la substitution d'un type d'espace à l'autre. A l'espace faiblement différencié du milieu du XIX ${ }^{\text {ème }}$ siècle succède le partage entre espaces dynamiques exerçant une forte pression sur le milieu et sur la ressource en eau et espaces fragiles, peu peuplés, couverts d'herbages et de forêts. Ces espaces correspondent pour l'essentiel aux zones de formation des débits du faisceau qui va de la Loire à la Vienne en englobant l'Allier, le Cher et l'Indre. Resterait à établir la relation entre ce type d'espace et la qualité de l'eau qu'il fournit aux bas pays environnants.

\subsubsection{Un tissu industriel assez lâche mais assez uniformé- ment réparti}

A l'échelle du bassin, le taux d'emploi industriel est égal à la moyenne française et il n'existe aucune concentration industrielle comparable à celles du Nord de la France, de la Basse-Seine ou de la région lyonnaise. Par contre, il n'existe à l'échelle du bassin ligérien aucune région dépourvue d'activités industrielles, alors qu'il est généralement admis qu'à une phase pré-industrielle caractérisée par la dissémination spatiale des activités manufaturières, a succédé une phase de concentration favorisant d'abord les régions d'extraction et de première transformation, puis les grands axes et les carrefours. Au vu des cartes de rejets industriels, ce schéma demande à être sérieusement révisé dans le cas du bassin ligérien. Quelle que soit la nature du rejet envisagé, matières organiques, phosphore ou substance inhibitrice, on observe bien une concentration correspondant à l'axe ligérien avec des zones fortes comme Orléans, Tours, Angers et surtout Nantes, mais il existe bien d'autres concentrations sur les vallées affluentes comme celle de la Vienne ou sur les cours amont dans les régions de SaintEtienne et de Clermont-Ferrand.

De nombreux facteurs expliquent cette dissémination dont le plus évident est la tradition hercynienne - charbon et forges - qui a profondément marqué Saint-Etienne et Montluçon : on n'exploite plus le charbon mais beaucoup d'activités relevant de la sidérurgie et de la mécanique sont restées. Ailleurs, à Roanne mais aussi dans la Mayenne, c'est la tradition textile et son cortège d'industries chimiques qui font encore sentir leur influence. Le long de la Vienne, ce sont les industries du feu, du cuir et du bois avec d'étonnantes relances comme celle de la papeterie de Saillat. Toutes ces activités ne relèvent pas forcément de la tradition, à preuve la présence de Michelin à Clermont-Ferrand. Enfin, les industries agro-alimentaires sont présentes sur toute l'étendue du bassin, souvent par le biais de petites installations qui n'ont pas toujours les moyens de traiter correctement leurs effluents. Dernier élément à prendre en compte, beaucoup d'activités révolues laissent après leur disparition des héritages encombrants, surtout s'il s'agit d'activités minières : la référence à Bessines-sur-Gartempe s'impose, mais il ne faut pas oublier qu'il existe bien d'autres sites à risques, certains datant du Moyen-Age.

Cette omniprésence de l'industrie pose d'évidents problèmes, aggravés par le fait que la plupart des activités de transformation, en dehors de l'axe ligérien proprement dit, relèvent de branches stagnantes ou récessives. Pour quelques secteurs dynamiques disposant des moyens nécessaires à l'épuration de leurs rejets et pour de nombreuses municipalités soucieuses du traitement de leurs rejets industriels, il existe donc, surtout dans les vieilles régions industrielles en perte de vitesse, des lacunes flagrantes au niveau de l'équipement. La qualité des eaux venues d'amont s'en ressent.

\subsubsection{La montée des loisirs}

Il est difficile de déterminer la part de l'eau dans la fréquentation touristique du Val de Loire. Le fleuve et ses 
bancs de sable font partie du décor, sans qu'il soit toujours possible d'en évaluer la perception en dehors des «parcours obligés» comme la terrasse de Chaumont («belle vue sur le village et le fleuve» précisent les guides autorisés) ou les arches de Chenonceau. L'eau est également partie prenante dans un grand nombre de sites remarquables, intégrant aussi bien les sites naturels comme le site Corot sur la Gartempe, que des sites artisanaux comme les moulins de la Sarthe ou celui de Richard-le-Bas, des sources et stations thermales comme Vichy.

De façon plus diffuse mais non moins importante, la pêche anime de nombreux parcours et suscite un volume d'activités non négligeable, à travers l'alevinage, l'entretien des berges et les indispensables établissements de services. C'est toutefois un loisir en stagnation si ce n'est en déclin. C'est également un loisir conflictuel, dans la mesure où l'entente est rarement parfaite entre pêcheurs et sportifs (canoë-kayak, radelage) ou baigneurs, les uns et les autres étant d'ailleurs honnis par les naturalistes.

Au titre des formes de loisir de l'eau en progression, il convient de porter les étangs, étangs de pêche ou de baignade qui font maintenant partie de l'équipement touristique de base, surtout sur les communes qui sont éloignées de tout cours d'eau. Là encore, le phénomène peut prendre une dimension conflictuelle, surtout lorsqu'il intéresse les ouvrages de grande hydraulique. Le cas de Villerest est assez caractéristique : les communes riveraines y voient des sites d'implantation pour les loisirs de l'eau y compris la planche à voile, alors que la logique d'exploitation veut que la saison chaude coïncide avec une phase de vidange. Il existe même un cas de détournement manifeste à Vassivière, vaste plan d'eau destiné à soutenir les étiages de la Vienne et à permettre le refroidissement de la future centrale de Civaux. Mais pour l'essentiel, Vassivière est devenu un parc de loisirs proche de Limoges, cerné de guinguettes et de bases nautiques, exploité par une compagnie de navigation commerciale et même pourvu d'un musée. Tout cela ne semble guère compatible avec les exigences du nucléaire.

\section{L'EAU ET LA STRUCTURATION DE L'ESPACE}

Dans quelle mesure et par quels vecteurs, l'eau contribuet-elle à l'organisation de l'espace ? La réponse n'est pas évidente tant sont nombreux les facteurs en cause et il faut convenir d'entrée de jeu qu'il n'existe pas, comme on l'a cru un temps, de «loi de l'eau». Du moins est-il possible de faire état des contraintes et des choix les plus évidents en opérant les distinctions qui s'imposent entre diverses échelles d'espace.

\subsection{Contraintes}

Au registre des contraintes ou si l'on préfère des transgressions, le problème essentiel est celui des plaines inondables. Le Val constitue bien entendu un cas spécifique, puisque son lit majeur est délimité par les levées et que l'espace correspondant au champ d'inondation a été sous- trait à son emprise. Les transgressions sont également minimes et de faible incidence dans les secteurs de roches cristallines. Il n'en va pas de même sur de nombreux petits cours d'eau de plaine, frangés par de minces rideaux d'arbres destinés à stabiliser leurs berges (peut-on alors parler de ripisylve ?). Leurs champs d'inondation ont été longtemps occupés de façon discrète par des prairies mais depuis quelques années, la priorité accordée aux grains par la politique agricole européenne et les facilités d'arrosage que confèrent à ces terres la proximité de l'eau, font que les labours tiennent maintenant une place excessive. Labourées et ameublies mais non pas protégées, ces terres sont sujettes à érosion à la moindre submersion. Elles sont de plus fertilisées et la percolation rapide des engrais et pesticides constitue un problème dont l'importance n'est pas toujours perçue.

Se posent également les problèmes des villes, avec les classiques inondations de bas quartiers souvent dévolus à des entrepôts ou des établissements industriels. En dépit de la protection qu'assurent les levées, c'est tout de même dans le Val que se posent les problèmes les plus sérieux en raison de la combinaison fâcheuse résultant du rétrécissement au niveau des vieux ponts et de la végétalisation croissante du Val. Les grands arbres y étaient rares autrefois, car on coupait du bois de chauffe entre les levées. Mais depuis une quarantaine d'années, les arbres ne sont plus coupés et il leur arrive d'être déchaussés et de partir au fil de l'eau. Par contre, des peupleraies apparaissent entre les levées. Que se passera-t-il le jour où une arche de pont sera obstruée par un chablis en temps de crue?

\subsection{Suggestions du milieu et choix des hommes}

A l'échelle la plus modeste, les alvéoles granitiques ont longtemps fonctionné et fonctionnent encore dans certains cas comme des lieux de vie. Une petite usine constitue souvent, sur la Gartempe ou sur la Creuse, la seule source d'emploi pour un petit village et, en dépit de la relance de la petite hydraulique, la raréfaction de ces témoins du passé pèse lourdement sur la destinée de nombreuses vallées du Massif Central. A un niveau plus élevé, ce ne sont pas des villages mais des petites villes qui vivent des activités liées à l'eau : c'est, pour peu de temps encore, le cas de BrivesCharensac ; c'est aussi le cas de Thiers qui a su trouver des relais à la coutellerie traditionnelle ; c'est enfin le cas d'une bonne dizaine de villes d'eau.

A l'échelle des réseaux urbains, le bassin ligérien présente une situation originale. Le problème du franchissement de la Loire par des gués ou des ponts a fixé le site de nombreuses villes qui commandaient les passages entre le Nord et le Sud de la France. D'où la mise en place d'un dispositif maillé avec un axe urbain dominant, la Loire entre Nevers et Nantes, et des relais de villes correspondant aux vallées et passages vers le Sud. Ce dispositif adapté à des temps où la voie d'eau supplantait les chemins de terre et où tous ceuxci se valaient par leur égale médiocrité, n'a pas résisté à l'évolution des techniques de communication. Le Massif Central est apparu très tôt, et en dépit d'un équipement ferroviaire très volontariste, comme un obstacle qu'il valait 
mieux contourner par l'est et Lyon ou par l'ouest et Poitiers, qu'affronter. Les grandes villes de l'intérieur, Limoges, Clermont-Ferrand et Saint-Etienne ne commandent donc que des passages secondaires et n'ont pas joué le rôle de relais et de carrefour qui leur était initialement dévolu. De son côté, l'axe ligérien n'a pas donné lieu à l'émergence d'une grande métropole. La route de Paris était trop facile, et Orléans trop proche de la capitale pour que naisse au point d'inflexion du fleuve, une métropole disposant d'un fort commandement.

Les villes ligériennes sont donc plus nombreuses que de grande importance. Elles commandent des réseaux de faibles dimensions et peu hiérarchisés. L'analyse des annuaires spécialisés montrent qu'une seule ville, Nantes, propose des services de niveau international, sans doute en raison de sa fonction portuaire. Inversement, les enquêtes faites auprès des usagers révèlent une forte attraction sur l'espace ligérien, de métropoles qui lui sont étrangères : Paris, de façon écrasante et, dans une moindre mesure Bordeaux et Lyon. Faut-il pour autant soutenir avec Y. Babonaux $^{4}$ que l'espace ligérien est un espace marginalisé? Certes non, tant il est difficile d'assimiler l'axe ligérien à une marge, mais espace éclaté et cela de tout temps. La limite entre langue d'oïl et langue d'oc court de Limoges au Mont Pilat et bien d'autres limites culturelles tranchent dans l'espace ligérien, qu'il s'agisse du modèle des charrues traditionnelles, du toit des maisons ou de la référence au droit romain contre le droit coutumier. La dispersion des réseaux urbains s'inscrit donc dans la tradition tout comme la lenteur de la mise en chantier d'un réseau autoroutier désenclavant la partie méridionale du bassin.

\section{APPROCHE D'UNE GESTION INTÉ- GRÉE}

Cet éclatement des réseaux et des infrastructures, cette juxtaposition de régions qui communiquent plus avec Paris qu'entre elles, n'a été rompue un temps que par l'activité de la batellerie ligérienne. Mais quel relais notre époque pourrait-elle inventer ? La gestion de la ressource en eau à l'échelle du bassin n'est pas réellement motivante, quels que soient les efforts de l'EPALA dans ce domaine. C'est pourtant en fonction de l'eau que se posent un grand nombre de problèmes mais les conflits et les concurrences áutour de la ressource sont plus évidents que les solidarités. Ce phénomène n'est pas spécifiquement ligérien et on le retrouverait sur d'autres fleuves, mais il atteint ici une intensité qui ne peut aller qu'en s'affirmant en raison de la spécificité de la Loire qui appelle de toute évidence une gestion intégrée qu'imposent et complexifient l'irrégularité des débits, les risques inhérents à cette irrégularité et la nécessité de préserver les valeurs paysagères, patrimoniales et environnementales. C'est dans cette perspective difficile qu'a été réalisée une approche écosystémique étendue à l'ensemble du bassin, en vue de la définition d'un plan de gestion intégrée.

${ }^{4} \mathrm{Y}$. Babonaux : L'espace ligérien, in Ph. Vigier ed, scientif. : Une histoire de la Loire, 1986, Paris, Ramsay ed., 413p.
La méthode consiste à délimiter des régions homogènes d'une part au plan de l'environnement ${ }^{5}$ - les déterminants relevant dans ce domaine, du climat, du substrat géomorphologique, de la biomasse et de l'hydrologie - d'autre part au plan de la démographie et des activités socio-économiques, puis à confronter les deux registres d'analyse en vue de déterminer des zones d'action présentant des caratéristiques communes et posant des problèmes de même ordre en matière de gestion. La totalité de la surface du bassin a été traitée mais tous les critères convergent vers la gestion de la ressource hydrique. Ces critères retenus étant relativeent nombreux - 164 items allant de la définition des aquifères à l'inventaire des ouvrages hydrauliques en passant par toute la gamme des activités - et leur manipulation se faisant aussi bien à l'échelle des communes qu'à celle des bassins hydrographiques, il a été nécessaire de recourir à un système d'information géographique implanté sur logiciel ArcInfo. Les résultats ont été présentés sous forme de cartes élémentaires dont la manipulation a permis d'établir des cartes de synthèse, puis de proposer une partition de l'espace entre huit grandes régions et vingt régions de second niveau dont certaines ont dû être subdivisées en raison de la forte individualisation de certaines unités comme les Limagnes ou les régions d'étangs (Fig.1). Les vingt-sept unités retenues constituent un effectif raisonnable au plan de la gestion d'un ensemble couvrant plus de $100000 \mathrm{~km}^{2}$.

De cette recherche ressort tout d'abord l'intérêt et surtout la possibilité d'intégrer logiquement la totalité des données impliquant les activités humaines dans leur cadre environnemental, ce qui ne signifie pas qu'il existe pour autant des relations directes de cause à effet entre ce cadre et ces activités : si le modelé des régions cristallines du Massif Central (château d'eau occidental et oriental) et des marges armoricaines présentent de nombreuses analogies et si ces deux régions accordent la priorité aux herbages et à l'élevage, elles s'avèrent dissemblables tant sur le plan de la démographie que sur celui des résultats économiques : les hautes terres du Massif Central ont perdu en un siècle l'essentiel de leurs effectifs et leur population vieillit de façon inquiétante, alors que l'Ouest est à la fois plus jeune et plus densément peuplé ; les pacages l'emportent sur les labours dans le Massif central tout en reculant devant la friche et la forêt, alors que les cultures sarclées et les prairies artificielles attestent du dynamisme de l'Ouest ; l'atonie ou le dynamisme des villes petites et moyennes renforcent cette opposition et amènent à opérer le partage entre des régions fragilisées qui incluent outre les hautes terres du château d'eau oriental (à l'exception de quelques régions comme les Limagnes) et du château d'eau occidental, ainsi que les régions d'étangs (Brenne et Sologne), et les régions dynamiques, qu'elles soient à peu près vides d'hommes comme la Beauce ou qu'elles concentrent l'essentiel des hommes et des activités comme l'axe ligérien et surtout la région nantaise.

5 Cf. J.G.Wasson et J. Bethemont, Les structures régionales du Bassin de la Loire, dans cette même publication. 


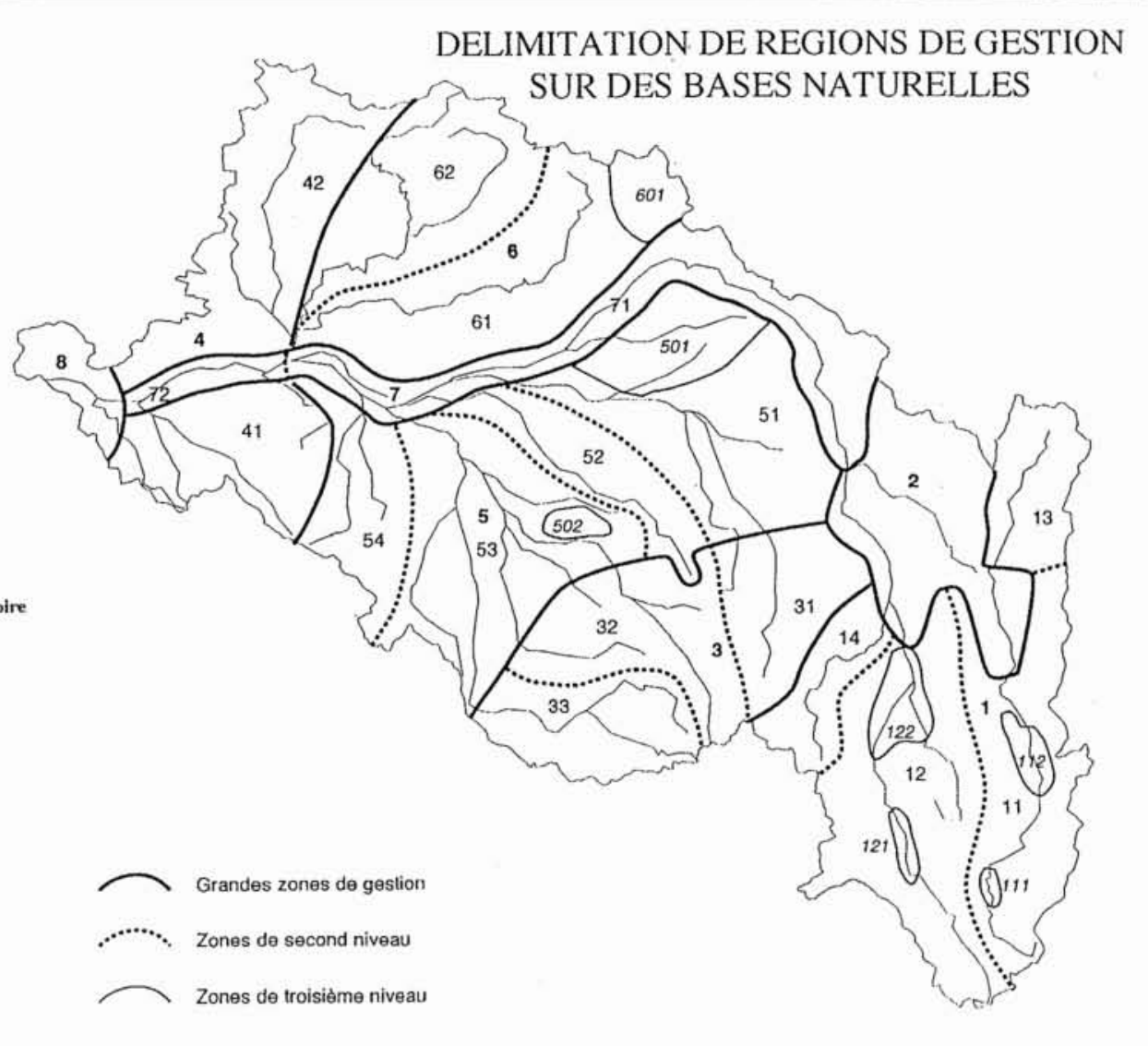

1. Délimitation de régions de gestion sur des bases naturelles.

Peut-on à partir de ce schéma, ramener les hautes terres du Massif Central au simple rôle de fournisseur d'une eau de plus en plus pure du fait de l'éradication progressive des activités humaines et peut-être d'une eau mieux régulée grâce à la progression de la forêt ? Au fil des enquêtes sur le terrain, il nous est apparu que les décideurs et les groupes de pression de l'axe ligérien n'étaient pas toujours hostiles à un tel scenario. L'impression que laisse dans certaines régions la fermeture progressive des paysages par la reforestation ou la suppression des chemins vicinaux, amène à réagir contre ce type de position. La prise en compte des efforts consentis par la fraction jeune et dynamique des populations de ces hautes terres pour valoriser leurs terroirs, tant par le maintien des activités traditionnelles que par le développement de nouvelles activités allant de la micromécanique au tourisme vert, va dans le même sens. S'il est certain que les hautes terres fonctionnent comme des châteaux d'eau et appellent de ce fait une gestion prudente en vue du maintien qualitatif de la ressource hydrique, il semble que ce type d'objectif ne soit pas incompatible avec le maintien des hommes et de leurs activités. C'est dans cette perspective que pourraient être définis les termes d'une gestion globale impliquant une réelle solidarité ligérienne, sur la base d'un principe simple mais d'application difficile : les régions amont fournissent une eau dont la qualité est maintenue ou améliorée par des aides et des investissements sélectifs en provenance des régions aval. Reste qu'à ce jour, un appel à la solidarité amont-aval relève de l'utopie. 